\title{
Síndrome de Panayiotopoulos. Cuadro clínico y alteraciones electroencefalográficas
}

\section{Panayiotopoulos syndrome. Clinical manifestations and electroencephalographic features}

\author{
Jorge Alderete Berzabá, ${ }^{*}$ David Islas García, ${ }^{\ddagger}$ Claudia Vannesa Quiroz Serna, ${ }^{\S}$ Rilma Nelly Perera Canul§
}

Citar como: Alderete BJ, Islas GD, Quiroz SCV, Perera CRN. Síndrome de Panayiotopoulos. Cuadro clínico y alteraciones electroencefalográficas. An Med (Mex). 2021; 66 (1): 67-71. https://dx.doi.org/10.35366/99493

\section{RESUMEN}

El síndrome de Panayiotopoulos es una epilepsia benigna de la infancia, diagnosticada comúnmente entre los tres y seis años, que se caracteriza por manifestaciones autonómicas, síntomas visuales y/o crisis motoras focales simples. La etiopatogenia aún no es clara. Se ha postulado la activación de áreas de bajo umbral de estructuras límbicas del cerebro interconectadas al sistema nervioso autónomo. Como hallazgos electroencefalográficos, se presentan puntas occipitales y extraoccipitales de gran amplitud. Las crisis son autolimitadas, con remisión espontánea uno o dos años después del inicio de la sintomatología. Objetivo: Describir las alteraciones clínicas y electroencefalográficas características en síndrome de Panayiotopoulos. Reporte de caso: Presentamos a un niño que inicia padecimiento a los 18 meses con convulsiones febriles tónico-clónicas generalizadas. A los cuatro años presenta palidez generalizada, náusea, vómito, seguido por versión cefálica y ocular a la izquierda, de tres a cinco minutos

\section{ABSTRACT}

Panayiotopoulos syndrome is a benign epilepsy of childhood, commonly diagnosed between 3 and 6 years of age, characterized by autonomic manifestations, visual symptoms and/or simple focal motor attacks. The etiopathogenesis is not all clear. It has been postulated that the activation of low threshold areas of limbic structures of the brain interconnected to the autonomic nervous system. As electroencephalographic findings, high amplitude occipital and extraoccipital spikes are presented. Seizures are self-limited, with spontaneous remission one or two years after the onset of symptoms. Objective: Description of the clinical and electroencephalographic features in Panayiotopoulos syndrome. Case report: We presented an 18-month-old baby present with clonic tonic febrile seizures. At age four years old, he presented generalized pallor, nausea, vomiting, followed by cephalic and ocular version to the left, lasting three to five minutes. Neurological examination and neuroimaging were normal. Electroencephalogram showed
* Médico Especialista en Neuropediatría. Subespecialidad en Neurofisiología Clínica. Adscrito al Servicio de Neurofisiología Clínica.

‡ Médico Especialista en Neuropediatría. Adscrito al Servicio de Neurología Pediátrica.

$\S$ Médico Especialista en Medicina de Rehabilitación. Médico Residente del segundo año de la Subespecialidad en Neurofisiología Clínica.
Correspondencia: Dr. Jorge Alderete Berzabá

Centro Neurológico del Centro Médico ABC Campus Santa Fe Av. Carlos Fernández Graef Núm. 154,

Santa Fe, Contadero, 05330.

Cuajimalpa de Morelos, CDMX.

E-mail: jorgeberza@hotmail.com

Abreviaturas:

$\mathrm{SP}=$ Síndrome de Panayiotopoulos. $\mathrm{EEG}=$ Electroencefalograma. 
de duración. La exploración neurológica fue normal al igual que resonancia magnética de encéfalo. Electroencefalograma anormal con puntas y ondas agudas en regiones occipitales bilaterales facilitadas en sueño. El trazo electroencefalográfico y la clínica corresponde a síndrome de Panayiotopoulos. Se inicia tratamiento con carbamazepina y levetiracetam. El pronóstico en general es favorable. El patrón electroencefalográfico focal puede modificarse de acuerdo con el tiempo de evolución. Es importante hacer un diagnóstico electroclínico para optimizar la incidencia de la enfermedad.

Palabras clave: Síndrome de Panayiotopoulos, puntas occipitales, electroencefalograma. spikes and sharp waves over both occipital regions mainly during sleep, consistent with Panayiotopoulos syndrome. Antiseizure medication with carbamazepine and levetiracetam was started. The prognosis is generally favorable. The focal electroencephalographic pattern may change over time. It is important to make an electro-clinical diagnosis to evaluate the incidence of the disease.

Keywords: Panayiotopoulos syndrome, occipital spike, electroencephalogram.

\section{INTRODUCCIÓN}

El síndrome de Panayiotopoulos (SP) es una epilepsia benigna de la infancia, caracterizada por convulsiones con signos y síntomas ictales autonómicos, fue descrita por primera vez por Chrysostomos Panayiotopoulos en $1988 .{ }^{1}$ La prevalencia de SP es del $13 \%$ entre las epilepsias de inicio temprano y del $6 \%$ en niños entre uno y 15 años; no hay prevalencia de género. Ocurre entre uno y 14 años, el pico máximo es entre tres y seis años. La incidencia es de 2-3/10,000 niños. ${ }^{2}$ La fiebre es a menudo un desencadenante de convulsiones en $\mathrm{SP}^{1,3}$

El cuadro clínico se manifiesta comúnmente con la tríada característica de náuseas, arcadas y vómitos $^{4,5}$ con posterior desviación de la mirada conjugada y versión cefálica, asociadas a crisis motoras simples que pueden o no generalizarse. ${ }^{6,7}$

El diagnóstico se realiza a través de pruebas genéticas, hallazgos electroencefalográficos ${ }^{8}$ y descartando anomalías estructurales en la neuroimagen. ${ }^{9,10}$

En el electroencefalograma (EEG) se observan puntas y ondas agudas de alto voltaje multifocales, todas las regiones cerebrales pueden verse afectadas, pero la anormalidad más común es en las regiones posteriores. ${ }^{11}$ Los focos de EEG frecuentemente cambian, se multiplican y se propagan difusamente relacionados con la edad, ${ }^{12,13}$ en lugar de localizarse de manera persistente en las regiones occipitales; la migración del foco en el EEG ocurre por un proceso de maduración del cerebro llamado «síndrome de susceptibilidad a las crisis benignas infantiles», en el cual la actividad epileptiforme inicia en la región occipital y progresa a regiones frontocentrotemporales conforme va madurando el cerebro. ${ }^{14}$ La neuroimagen por resonancia magnética no muestra alteraciones significativas en SP; sin embargo, es útil para el diagnóstico diferencial. ${ }^{14,15}$

\section{CASO CLÍNICO}

Paciente masculino de nueve años, con antecedentes de restricción en el crecimiento intrauterino a partir de las 30 semanas de gestación, obtenido por vía abdominal a las 39 semanas de gestación por oligohidramnios, APGAR 7/9. Síndrome de aspiración de meconio con mejoría a los tres días y egreso hospitalario. Inicia padecimiento a los 18 meses de edad presentando crisis febriles tónico-clónicas, presenta crisis convulsiva nuevamente asociada a fiebre a los tres meses, se inicia tratamiento con carbamazepina.

A los cuatro años acude a nueva valoración médica, al estar libre de crisis por dos años se decide suspender manejo con carbamazepina; a los dos meses de haber suspendido el fármaco, presenta crisis convulsivas caracterizadas por palidez generalizada, náusea y vómito, seguido por versión cefálica y ocular a la izquierda de cinco minutos de duración por lo general al despertar o después de tomar siestas, padece además somnolencia en el postictal. Estas crisis se exacerban al presentar infecciones respiratorias, con una frecuencia de tres al año. La exploración neurológica fue normal, al igual que la resonancia magnética de encéfalo, EEG interictal anormal con brotes de ondas agudas y puntas en regiones occipitales bilaterales durante vigilia que se facilitan en sueño. El trazo de EEG y la clínica corresponden a síndrome de Panayiotopoulos, por lo que se decide reiniciar tratamiento con carbamazepina y se agrega levetiracetam por la exacerbación del cuadro clínico.

En la actualidad, se encuentra libre de crisis desde hace un año, aún con fármacos antiepilépticos. Con vigilancia clínica y electroencefalográfica, el último EEG hace un año persiste con anormalidades características de la enfermedad. 


\section{DISCUSION}

Se reporta el caso clínico típico de SP, los criterios para su diagnóstico son: 1) manifestaciones autonómicas, síntomas visuales y/o crisis focales motoras simples, seguidos por deterioro de la conciencia, con o sin generalización secundaria; 2) estado neurológico intercrítico normal; 3) neuroimagen normal; y 4) paroxismos difusos como la primera manifestación de EEG en el inicio clínico. ${ }^{6,13}$ Utilizamos los criterios de la liga internacional contra la epilepsia (ILAE por sus siglas en inglés) para establecer el diagnóstico de síndrome de Panayiotopoulos, cumpliendo con edad de inicio, exploración neurológica normal, crisis obligatorias de tipo focal autonómico principalmente gastrointestinal, así como patrón de electroencefalograma característico con puntas occipitales.

Las crisis son poco frecuentes en la mayoría de los casos con SP, en nuestro paciente se asociaron a infecciones y/o estado febril y fueron frecuentes. Sólo el 25\% tiene una crisis aislada (que puede ser estado epiléptico autonómico), y el 50\% tiene seis crisis o menos durante la evolución de la enfermedad $;^{3}$ se presume que la frecuencia excepcional de las crisis autonómicas en el SP se debe a un umbral bajo genéticamente determinado relacionado con la edad. ${ }^{1,7}$ Tiene un buen pronóstico, las crisis son autolimitadas y vuelven a aparecer, en general, dentro de uno a dos años desde la presentación inicial. En algunos casos se suelen resolver hasta entre los 11 y 13 años; no se reportan alteraciones significativas del desarrollo neurocognitivo. . $^{1,5,10}$

Los reportes neuropsicológicos demuestran sutiles deficiencias en el lenguaje y el funcionamiento ejecutivo, así como afección de la memoria visual y problemas de lectura durante los periodos de crisis activas. ${ }^{11,14} \mathrm{El}$ estado epiléptico convulsivo motor es extremadamente raro; sin embargo, el estado epiléptico autonómico es muy común, consistiendo en crisis prolongadas con duración mayor de 30 minutos a un par de horas (aproximadamente dos horas) con pérdida de conciencia. ${ }^{10}$ Se especula que la prolongación de las crisis autonómicas sea debida a la interacción entre los sistemas simpático y parasimpático, como entre las funciones recíprocas de las estructuras centrales y los órganos terminales, que tienen un efecto competitivo y que podría influir negativamente en los mecanismos de terminación de estas crisis autonómicas. ${ }^{7}$ Las convulsiones parecen ser el producto de una excitación directa de la neocorteza y las áreas corticales límbicas con la posterior propagación a las estructuras de la red autonómica central. ${ }^{1}$ La exci- tabilidad del sistema autónomo, independiente del punto de partida de la actividad, es frecuentemente occipital. $^{7}$

Se puede provocar hiperactivación del sistema nervioso autónomo a través de la red autonómica central (CAN). El CAN es un circuito extenso que incluye la corteza insular, la amígdala, el hipotálamo, la materia gris periacueductal, el complejo parabraquial, el núcleo del tracto solitario y la médula ventrolateral; controla las respuestas visceromotoras, neuroendocrinas, de dolor y conductuales, y es inmadura en los niños. Según esta hipótesis, el umbral de activación de la sintomatología autonómica puede ser menor en CAN que en las áreas motoras o sensoriales. Este umbral inferior podría explicar la razón por la cual una descarga epiléptica activa las redes autonómicas, causando síntomas de dicha naturaleza sin el reclutamiento de áreas corticales vecinas, que controlan las manifestaciones ictales motoras o sensoriales. Después de una breve latencia, el foco epiléptico propaga a corteza cerebral (sensorial-motora, parietal, occipital), que muestra un umbral relativamente más alto, lo que genera síntomas focales. Esta hipótesis puede explicar las características autonómicas pronunciadas de la condición. ${ }^{1}$

Dado que el SP tiene un buen pronóstico, no se recomienda una profilaxis antiepiléptica, el pronóstico no parece ser modificado por la terapia farmacológica antiepiléptica continua; el tratamiento se requiere sólo para niños en quienes las convulsiones son inusualmente frecuentes o interfieren de manera significativa en la calidad de vida, ${ }^{10}$ además, los casos con convulsiones aisladas no deben ser tratados con anticonvulsivos. ${ }^{6}$ Debido a las múltiples crisis que nuestro paciente presentó a edades tempranas, se decidió iniciar manejo con monoterapia. Algunos autores sugieren utilizar carbamazepina, ácido valproico o fenobarbital después de la segunda convulsión, dependiendo de la duración de las convulsiones y la presencia de anomalías neurológicas asociadas, en general, la duración total del tratamiento con fármacos antiepilépticos debe durar de dos a tres años, ${ }^{1}$ también es útil eliminar los desencadenantes de las convulsiones: drogas, alcohol y falta de sueño. Las alteraciones cardiacas, respiratorias y neurológicas del sistema autonómico promueven la muerte súbita e inesperada en la epilepsia (SUDEP por sus siglas en inglés), aunque la patogenia aún no es clara. ${ }^{2}$ Se pueden encontrar mutaciones en $\mathrm{SCN}_{1} \mathrm{~A}^{16}$ en algunos pacientes con SP y convulsiones febriles, las pruebas genéticas también pueden ayudar a establecer el pronóstico; en SUDEP se han identificado mutaciones 
codificadas por RYR2 y SCN5A para el canal de sodio Nav1.5 cardiaco. $^{1}$

Los hallazgos en el EEG describen anomalías predominantes en las regiones posteriores, ${ }^{1}$ las puntas multifocales de alto voltaje repetitivas y ondas lentas agudas se ven en el $90 \%$ de los pacientes. ${ }^{3}$ Las características del EEG en SP sugieren un umbral epileptogénico bajo único que involucra circuitos cerebrales particulares con vulnerabilidad dependiente de la edad. ${ }^{15}$

Existe una gran variabilidad electroencefalográfica de la localización de las puntas focales, las puntas occipitales bilaterales y sincrónicas son las anormalidades más comunes descritas en SP, a menudo asociadas con puntas extraoccipitales concurrentes (60\%); las puntas frontales en los EEG de los pacientes con SP se describieron como una activación secundaria desencadenada por descargas occipitales, lo que sugiere propagación posteroanterior, y esta propagación de la actividad epiléptica forma una red extensa en SP. ${ }^{17,18}$

Los casos con un patrón de EEG generalizado interictal tienen convulsiones más frecuentes y una duración significativamente mayor del periodo de crisis activa que otros con patrón de EEG focal. ${ }^{10} \mathrm{La}$ actividad de fondo es normal (Figura 1A), se observan puntas repetitivas y ondas agudas de alto voltaje multifocales, ${ }^{3}$ todas las regiones pueden ser afectadas. ${ }^{6,10}$ En nuestro paciente, la mayoría de EEG demuestran una anormalidad persistente en región occipital (Figura 1B). Los focos de EEG suelen cambiar, se multiplican y se propagan de forma difusa relacionados con la edad, ${ }^{2,18}$ en lugar de localizarse en las regiones occipitales; en particular, la migración del foco epileptógeno en EEG desde la región occipital a las frontocentro-temporales parece ser un proceso de maduración del cerebro llamado «síndrome de susceptibilidad a las crisis benignas infantiles», ${ }^{10}$ mismo desafío que enfrentaron los primeros electroencefalografistas de una localización poco precisa de las redes epileptogénicas en casos de epilepsia focal. ${ }^{19}$ Las lentificaciones generalizadas sólo se ven en periodos postictales. ${ }^{20}$

El cierre de los ojos (eliminación de la visión central) puede activar las puntas occipitales, las cuales se bloquean al abrir los ojos (Figura 1C). En sueño ${ }^{3}$ esta actividad tiende a tener un mayor campo y pueden ser bilateralmente sincrónicas (Figura 1B). La estimulación fótica intermitente suele ser negativa, pero se puede observar una respuesta fotoparoxística que consiste en complejos generalizados de punta $\mathrm{u}$ onda aguda con gran amplitud sobre las regiones occipitales. ${ }^{1}$ La amplitud de las ondas agudas fue notablemente mayor en la etapa NREM y se extienden principalmente a las regiones central y centrotemporal. ${ }^{7}$ Los patrones ictales son unilaterales, a menudo tienen un inicio posterior con actividad rítmica lenta (theta o delta) entremezclada con puntas. ${ }^{3,10}$ En el EEG, las pendientes de las descargas epileptiformes interictales son a menudo asimétricas con el componente negativo inicial típicamente más pronunciado, seguido de un componente positivo más lento. ${ }^{4}$

Se observan puntas occipitales con frecuencia en la epilepsia occipital infantil (Panayiotopoulos y Gas-

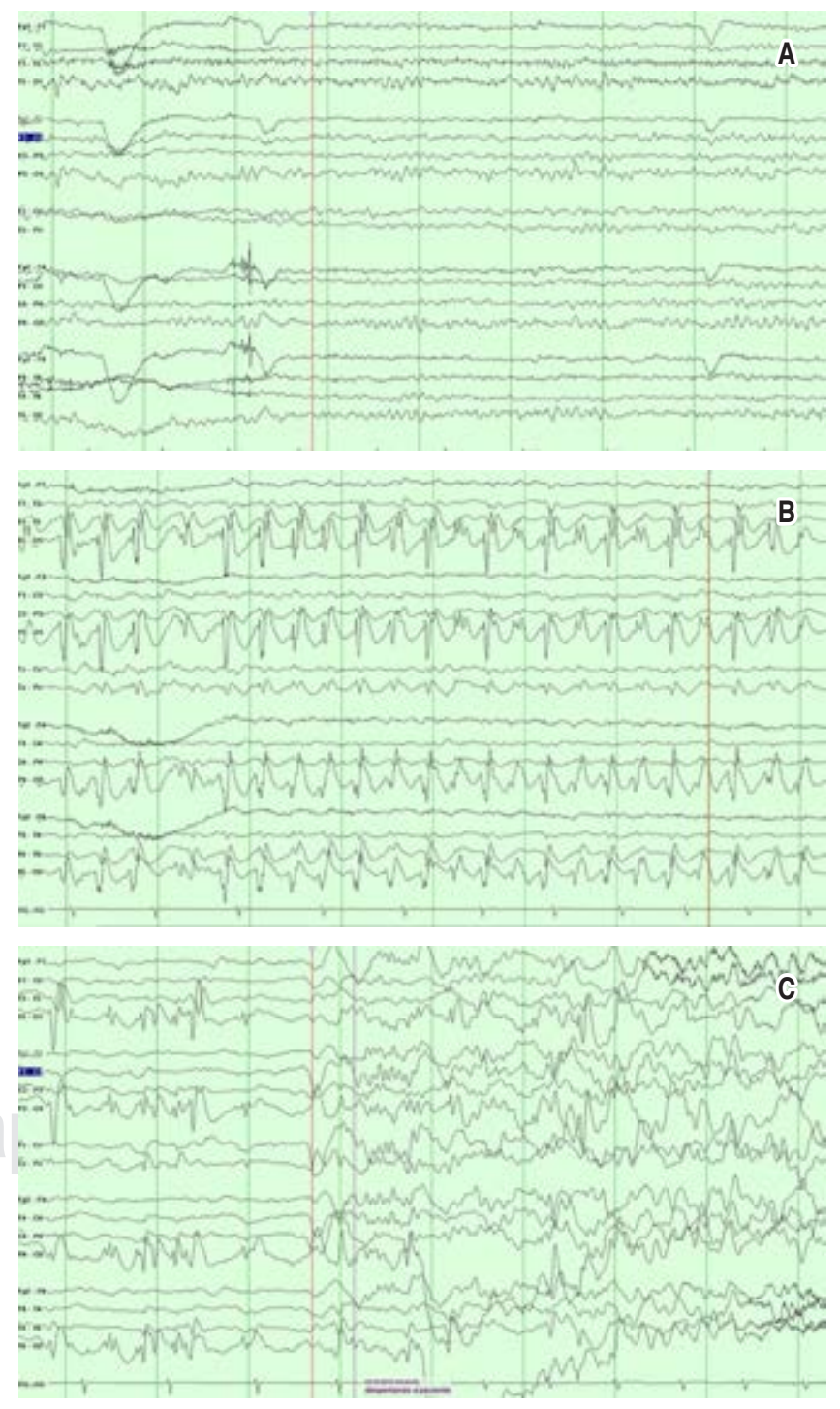

Figura 1: Registro interictal. A) Vigilia, actividad de fondo en rango alfa de 8 a 9 Hz. B) Ondas agudas occipitales en somnolencia. C) Ondas agudas bloqueadas al abrir los ojos. 
taut), así como en otros tipos de epilepsia del lóbulo occipital, ${ }^{8}$ las cuales presentan síntomas visuales en su mayoría, como alucinaciones. ${ }^{1,9}$ Las crisis focales que dan lugar a alucinaciones visuales raramente progresan en áreas corticales extravisuales, y a menudo se asocian a una cefalea postictal que no se puede distinguir de la migraña. ${ }^{12,14}$

También se puede hacer diagnóstico diferencial de SP con epilepsia rolándica benigna, caracterizada por descargas EEG focales en las áreas centrales y centrotemporales, con edad de aparición máxima de tres a seis años, muy similar a SP. ${ }^{5}$ Se ha reconocido ampliamente que las epilepsias focales idiopáticas de la infancia y las epilepsias generalizadas idiopáticas pueden coexistir, ya sea al mismo tiempo u ocurrir en diferentes periodos de edad en la misma persona. ${ }^{6}$

Trastornos agudos no epilépticos como el síndrome de vómito cíclico, la gastroenteritis y reflujo gastroesofágico (ERGE) son otros de los diagnósticos a considerar debido a que los síntomas eméticos son las características clínicas predominantes al inicio de las convulsiones en SP. ${ }^{1}$

Las crisis desaparecen después de un año del inicio, a pesar de las anormalidades persistentes de EEG. ${ }^{6}$

\section{CONCLUSIÓN}

Este caso ilustra el cuadro clínico y electroencefalográfico característico de SP, el cual puede estar relacionado con una hiperexcitabilidad cortical extensa genéticamente determinada, que involucra un sistema específico del cerebro. Este concepto explica los hallazgos electroclínicos focales y difusos en niños con SP. La epilepsia rolándica benigna tiene una frecuencia de presentación ocho veces mayor que SP, por lo que es necesario considerar que los hallazgos electroencefalográficos se modifican con la edad, para evitar así la confusión de ambos síndromes; teniendo en cuenta que son manifestaciones principalmente autonómicas, se debe esperar que haya mayor incidencia de SP.

\section{REFERENCIAS}

1. Graziosi A, Pellegrino N, Di Stefano V, Raucci U, Luchetti A, Parisi P. Misdiagnosis and pitfalls in Panayiotopoulos syndrome. Epilepsy Behavior. 2019; 98: 124-128.

2. Oguni H, Hirano Y, Nagata S. Encephalopathy related to status epilepticus during slow sleep (ESES) as atypical evolution of Panayiotopoulos syndrome: an EEG and neuropsychological study. Epileptic Disord. 2020; 22 (1): 67-72.
3. Panayiotopoulos syndrome [Internet]. Epilepsydiagnosis. org. 2020 [Cited 2 June 2020]. Available from: https://www. epilepsydiagnosis.org/syndrome/panayiotopoulos-videos.html.

4. Chen H, Koubeissi M. Electroencephalography in epilepsy evaluation. Continuum (Minneap Minn). 2019; 25 (2): 431-453.

5. Weir E, Gibbs J, Appleton R. Panayiotopoulos syndrome and benign partial epilepsy with centro-temporal spikes: a comparative incidence study. Seizure. 2018; 57: 66-69.

6. Caraballo R, Pasteris M, Portuondo E, Fortini P. Panayiotopoulos syndrome and diffuse paroxysms as the first EEG manifestation at clinical onset: a study of nine patients. Epileptic Disord. 2015; 17 (2): 143-149.

7. Tata G, Guveli B, Dortcan N, Cokar O, Kurucu H, Demirbilek $\mathrm{V}$ et al. Panayiotopoulos syndrome and symptomatic occipital lobe epilepsy of childhood: a clinical and EEG study. Epileptic Disord. 2014; 16 (2): 197-202.

8. Michael M, Tsatsou K, Ferrie C. Panayiotopoulos syndrome: an important childhood autonomic epilepsy to be differentiated from occipital epilepsy and acute non-epileptic disorders. Brain Dev. 2010; 32 (1): 4-9.

9. Pelak V. Visual hallucinations and higher cortical visual dysfunction. Continuum: Lifelong Learning in Neurology. 2009; 15 (4): 93-105.

10. Parisi P, Villa M, Pelliccia A, Rollo V, Chiarelli F, Verrotti A. Panayiotopoulos syndrome: diagnosis and management. Neurol Sci. 2007; 28 (2): 72-79.

11. Ferrie C, Caraballo R, Covanis A, Demirbilek V, Dervent A, Kivity S et al. Panayiotopoulos syndrome: a consensus view. Dev Med Child Neurol. 2006; 48 (3): 236-240.

12. Galanopoulou A, Lado F. Classification, pathophysiology, causes, differential diagnosis of paroxysmal events. Continuum. Lifelong Learning in Neurology. 2004; 10 (4): 11-41.

13. Oreña-Ansorena VA, Blanco-Lago R, Hedrera-Fernández A, Bonifacio M, Santoveña-González L, Quesada-Colloto P et al. Epilepsia sintomática con inicio que imita el síndrome de Panayiotopoulos: importancia de la neuroimagen. Revista de Neurología. 2020; 70 (04): 134. Available from: https://doi. org/10.33588/rn.7004.2019493.

14. Akca KS, Elmali A, Demirbilek V, Oktem O, Yapici Z, Saltik $\mathrm{S}$ et al. Panayiotopoulos syndrome and Gastaut syndrome are distinct entities in terms of neuropsychological findings. Epilepsy \& Behavior. 2019; 99: 106447.

15. Saito N, Kanazawa O, Tohyama J, Akasaka N, Kamimura T, Toyabe $\mathrm{S}$ et al. Brain maturation-related spike localization in Panayiotopoulos syndrome: magnetoencephalographic study. Pediatr Neurol. 2008; 38 (2): 104-110.

16. Fujiwara T. Clinical spectrum of mutations in SCN1A gene: Severe myoclonic epilepsy in infancy and related epilepsies. Epilepsy Res. 2006; 70 (Suppl. 1): S223-230.

17. Leal AJ, Ferreira JC, Dias AI, Calado E. Origin of frontal lobe spikes in the early onset benign occipital lobe epilepsy (Panayiotopoulos syndrome). Clin Neurophysiol. 2008; 119 (9): 1985-1991.

18. Leal A, Lopes R, Ferreira J. Origin and dynamics of epileptic activity in a symptomatic case of Panayiotopoulos syndrome: correlation with clinical manifestations. Clinical Neurophysiology. 2013; 124 (1): 20-26.

19. Plummer C, Harvey A, Cook M. EEG source localization in focal epilepsy: Where are we now? Epilepsia. 2008; 49 (2): 201-218.

20. Panayiotopoulos CP. Vomiting as an ictal manifestation of epileptic seizures and syndromes. J Neurol Neurosurg Psychiatry. 1988; 51 (11): 1448-1451. 